\title{
Pensamiento Aritmético-Algebraico a través de un Espacio de Trabajo Matemático en un Ambiente de Papel, Lápiz y Tecnología en la Escuela Secundaria
}

\author{
Arithmetico-Algebraic Thinking through a Mathematical Working Space \\ in a Paper, Pencil, and Technological Environment in Secondary School
}

\author{
José Carlos Cortés* \\ Fernando Hitt $^{* *}$ \\ Mireille Saboya ${ }^{* * *}$
}

\begin{abstract}
Resumen
En este documento nos interesa abordar el problema de la enseñanza del álgebra bajo el punto de vista del marco teórico de los Espacios de Trabajo Matemático (ETM). En nuestro estudio, realizamos dos experimentaciones, una en Québec y otra en México. En la investigación, hemos considerando la visualización que se pone en marcha, una vez que el individuo comunica sus ideas a otros (o a sí mismo), y construye relaciones entre los elementos en juego, incluyendo articulaciones entre representaciones. La evolución de estas construcciones cognitivas dentro de una perspectiva de los procesos de significación, necesita de experiencias compartidas, en lo que nosotros hemos designado como una enseñanza y aprendizaje en colaboración, debate científico y autorreflexión (ACODESA), bajo el punto de vista de la teoría de la actividad. Específicamente, se investiga en torno a los números poligonales (en especial los números triangulares), en un ambiente de papel, lápiz y tecnología. Nuestra interpretación sobre los procesos cognitivos de una población de alumnos (14-15 años) de una escuela secundaria en México siguen el marco teórico de los ETM. Esta interpretación se centra en el paso de un plano epistemológico hacia un plano cognitivo en donde la actividad matemática permite una articulación de procesos de visualización, producción y procesos de validación aritmético-algebraicos que proporcionan un suporte precisamente a un pensamiento aritmético-algebraico.
\end{abstract}

Palabras Clave: ACODESA. Espacios de Trabajo Matemático. Pensamiento aritmético-algebraico.

\begin{abstract}
In this paper, we want to address the problem of teaching algebra from the point of view of the theoretical framework of Mathematical Working Spaces (MWS). In our study, we carried out two experiments, one in Quebec and one in Mexico. In this research, we consider the visualization has started, once the individual communicates his ideas to others (or himself) and builds relationships between the elements involved, including
\end{abstract}

\footnotetext{
* Doctorado en Matemática Educativa por el Cinvestav, México. Profesor Investigador Tiempo Completo en la Facultad de Ciencias Físico Matemáticas de la Universidad Michoacana, México. E-mail: jcortes@umich.mx. Edificio B, Ciudad Universitaria, Francisco J. Múgica S/N, Colonia Felicitas del Río, C.P. 58030, Morelia, Michoacán. México.

** Doctorado en Didáctica de las Matemáticas por la Universidad de Estrasburgo, Francia. Profesor Investigador en el Departamento de_Matemáticas de la Université du Québec à Montréal (UQAM). E-mail: hitt.fernando@uqam.ca. 201 Avenue Président Kennedy, Montréal, H2X 3Y7, Qc. Canada.

Doctorado en Educación por la Facultad de Educación de la UQAM. Profesora Investigadora del Departamento de Matemáticas de la Université du Québec à Montréal UQAM. E-mail: saboya.mireille@uqam.ca. 201 Avenue Président Kennedy, Montréal, H2X 3Y7, Qc. Canada.
} 
articulations among representations. The evolution of these cognitive structures within a perspective of the processes of objectification, requires shared experiences in which we have designated as a teaching and collaborative learning, self-reflection and scientific debate (ACODESA) under the point of view of activity theory. Specifically, it investigates polygonal numbers (especially triangular numbers) in an environment of paper, pencil, and technology. Our interpretation of students' (14-15 year old) cognitive processes of a population at secondary school in Mexico, following the theoretical framework of the ETM. This interpretation focuses on a step towards a cognitive epistemological plane, where mathematical activity enables articulation among visualization processes, production processes, and arithmetico-algebraic validation providing a support precisely to an arithmetico-algebraic thinking.

Keywords: ACODESA. Mathematical Working Spaces. Arithmetico-Algebraic Thinking.

\section{Introducción}

Nuestra investigación se centra en la importancia de generar un pensamiento articulado al pensamiento aritmético y al pensamiento algebraico. Realizamos experimentaciones en Quebec (alumnos de 12-13 años) y México (de 14-15 años). En este documento, nos restringiremos a la población de México, alumnos de $3^{\circ}$ de secundaria sobre la posibilidad de construir un pensamiento aritmético-algebraico lo suficientemente sólido, que les permita validar a los alumnos sus procesos de abstracción y generalización (aspectos que discutiremos más adelante) hacia un pensamiento algebraico, desde un apuntalamiento ligado al pensamiento aritmético y viceversa. De hecho, nuestra propuesta consiste en la construcción de un pensamiento aritmético - algebraico (de aquí en adelante lo denotaremos como PA-A) con estudiantes de secundaria. Para llegar a ello, primero analizaremos lo que en la literatura se entiende por pensamiento aritmético y por pensamiento algebraico, enseguida haremos referencia a la importancia de considerar una articulación entre dichos pensamientos y lo relacionaremos con los ETM, para considerar nuestra experimentación bajo este marco teórico, finalizando con una discusión sobre los resultados encontrados.

\section{1 ¿Qué es un pensamiento aritmético?}

Verschaffel y De Corte (1996), caracterizan el pensamiento aritmético en la escuela primaria como la estructura cognitiva que tiene que ver con:

- Conceptos numéricos y sentido de los números,

- El significado de las operaciones aritméticas,

- Control de hechos básicos de la aritmética,

- Cálculo mental y escritura de la aritmética,

- Lectura y escritura de problemas verbales y habilidades aritméticas. 
En cierta medida, podemos decir que la estructura promovida por Brownell (1942) ha prevalecido en el siglo pasado, él propuso que la inteligencia se debía medir en la resolución de problemas verbales aritméticos en el contexto escolar y no como los psicólogos de la época, que se centraban en intentar medir la inteligencia con problemas de tipo puzzle en donde el insight era el elemento principal para la resolución de ese tipo de problemas.

Regresando a Verschaffel y De Corte, podemos decir que estos autores implícitamente incluyen los procesos de abstracción en la resolución de problemas verbales; sin embargo, los procesos de generalización no parece que fueran considerados de manera explícita en la década de los 90s. La partición en esa época era muy clara, aritmética para primaria y el álgebra para secundaria. De hecho, esta tradición curricular impulsó un cierto tipo de investigación, centrada en la búsqueda de cortes entre la aritmética y el álgebra (FILLOY; ROJANO, 1989), o la noción de obstáculo epistemológico (BROUSSEAU, 1997) utilizada para dar cuenta de los problemas de aprendizaje del álgebra (VERGNAUD, 1990), entre otros.

Así, dado que los programas de estudio hacían una partición entre la aritmética y el álgebra, la investigación se desarrollaba de la misma manera, como lo mencionamos antes, algunos investigadores se dedicaron a analizar el pensamiento aritmético (p.e. VERCHAFELL; De CORTE, 1996 o VERGNAUD, 1990 en primaria), y otros el pensamiento algebraico en secundaria (KAPUT, 2000, 2008; FILLOY; ROJANO, 1989; KIERAN, 2007, entre otros). Bajo este punto de vista, era primordial para los investigadores determinar las diferencias entre un tipo de pensamiento. En la sección siguiente presentamos algunos trabajos en esta dirección, ligada al pensamiento algebraico (PA).

\section{2 ¿Qué es un pensamiento algebraico?}

De acuerdo a Kaput (2000, 2008), el pensamiento algebraico debe comportar en general dos aspectos principales:

1. Realizar y expresar la generalización de los sistemas simbólicos de manera más formal y convencional, $\mathrm{y}$

2. Razonar con formas simbólicas, incluyendo la manipulación sintáctica guiada de estas formas simbólicas.

Bajo este enfoque, Kaput nos propone considerar estos aspectos como etapas, bajo un punto de vista holístico, en la formación del pensamiento algebraico. Kieran (2007), por el contrario, intenta caracterizar de manera minuciosa los subconceptos que integran un 
pensamiento algebraico. Así, bajo el modelo de Kieran (2007, p. 714), podemos saber más sobre los aspectos locales del contenido a desarrollar en los estudiantes a través de su modelo GTG, que en resumen trata de:

G) La actividad generacional (por ejemplo, los patrones);

T) La actividad de transformaciones (factorización, expansión, ecuaciones equivalentes, etc.);

$\mathrm{G}_{\mathrm{m}}$ ) La actividad global / meta (resolución de problemas, modelado, justificación, reconocimiento de patrones, etc.)

Los trabajos de Kaput repercutieron fuertemente en el medio de investigación. Kaput, que tenía ya una tradición intentando promover conceptos del cálculo desde la escuela primaria, sobre variación y cambio, posiblemente tuvo un eco importante en su propuesta de proceso de desalgebrización que consistía en introducir ideas algebraicas en la escuela primaria. Ello promovió el llamado movimiento Early algebra.

Este movimiento (Early algebra) ha influido en cambios en los currículos en algunos países, por ejemplo en los de USA (ver CAI; KNUTH, 2011). Bajo esta línea, Artigue (2012), analizando las nuevas tendencias en los cambios curriculares con respecto a primaria y secundaria, opina que la entrada al pensamiento algebraico se puede realizar bajo diferentes caminos:

\begin{abstract}
En algunos países, es el recorrido histórico de las ecuaciones asociadas con el enfoque analítico cartesiano que se ha elegido, en otros, incluyendo la cultura anglosajona, es la forma de reconocimiento de patrones y la generalización que se ha seleccionado. Esto no intenta enseñar estructuras algebraicas sino identificar lo que se llaman patrones en secuencias de números, en configuraciones geométricas, para expresar algebraicamente y utilizar esta expresión algebraica para estudiar, caracterizar, comparar. En otros países, es el camino de modelación que se ha elegido, a menudo vinculados con situaciones extra-matemáticos. (p. 4).
\end{abstract}

Es así que el movimiento Early algebra ha promovido, entre los investigadores, el uso de patterns (podemos decir entre los investigadores más moderados) y el uso de series (un acercamiento funcional) sin pasar por los patterns (entre los investigadores que ven un pasaje más rápido hacia el álgebra). En el movimiento Early algebra se distinguen esas dos tendencias (ver CAI; KNUTH, 2011). Una tendencia moderada que investiga límites y potencialidades de aspectos sobre la abstracción y la generalización, digamos más centrada en aspectos estructurales de la matemática (CARPENTER; FRANKE, 2001; CARPENTER; FRANKE; LEVI, 2003; RADFORD, 2011; WARREN, 2006), y otros más entusiastas que van al extremo de promover un acercamiento algebraico en primaria desde una perspectiva funcional (CARRAHER; SCHLIEMANN; BRIZUELA; EARNEST, 2006; SCHLIEMANN; 
CARRAHER; BRIZUELA, 2012). En las secciones que siguen mostraremos nuestra posición con respecto a esas dos tendencias en educación matemática.

\section{Construcción de aspectos teóricos}

Nuestra intención en este documento, es el de utilizar la noción de Espacio de Trabajo Matemático, término acuñado por Kuzniak (2011). Sabemos que con un ejemplo basado en la geometría pero con la posibilidad de extenderlo a otras ramas, Kuzniak consideró un plano epistemológico y tres elementos relacionados a la abstracción: la intuición, la experiencia y la deducción. En términos epistemológicos, Kuzniak se refiere al espacio real y local dentro de un Espacio de Trabajo Geométrico (ETG) y a los artefactos repositorios que permiten a los alumnos generar procesos cognitivos en relación a la visualización, las matemáticas, la construcción y la prueba.

En un proceso de comparación e integración, discutimos enseguida lo que consideramos como representación institucional, representación funcional, visualización y generalización, con la finalidad de integrar estos constructos teóricos con el marco de los ETM de Kuzniak y con ello realizar posteriormente un análisis de los resultados de nuestra experimentación.

En nuestro estudio, nosotros le proporcionamos más importancia a las representaciones espontáneas producto de las representaciones funcionales (HITT, 2013; HITT; GONZÁLEZ-MARTÍN, 2015); que las que le proporciona Duval (1993, 1995, 2006). Así, en nuestro acercamiento, utilizaremos las siguientes definiciones:

- Representación institucional. Representaciones encontradas en libros de texto o en pantallas de ordenadores, o las que utiliza el profesor.

- Representación funcional. Representación mental que emana de la actividad matemática no rutinaria, que se expresa por una representación (representación espontánea) ligada a la acción.

- Visualizar. Es encontrar relaciones entre diferentes representaciones (no necesariamente institucionales) en un proceso de resolución de situaciones problema. Es decir, que nosotros incluimos las representaciones espontáneas que surgen en la resolución de problemas y de situaciones problema, por ello, estas representaciones no necesariamente pertenecen a un sistema específico de signos (p.e. un dibujo ligado a una situación) y además, consideramos su 
evolución en un medio de co-construcción sociocultural que tienden a transformarse, en un proceso de comunicación en el aula, en representaciones institucionales que son reconocidas en los registros de representación (ver HITT; GONZÁLEZ-MARTÍN, 2015).

- La generalización, en nuestro caso particular de este documento, tiene que ver con procesos de visualización y deducción que comprende aspectos intermedios de anticipación, conjetura y validación a partir de casos particulares.

Como lo señalamos antes, el currículo de matemáticas clásico hace una distinción entre aritmética y álgebra. Esta partición generó investigaciones para distinguir el pensamiento aritmético y el pensamiento algebraico. De acuerdo a la notación de Montoya y Vivier (2014) y a la explicación de Kuzniak (2014, p. 387) sobre el acercamiento de Chevallard (1999) sobre la organización de dominios, sería natural en pensar en dos ETM, el ETM $_{\text {Aritmético }}$ y el ETM algebraico y en la posibilidad de conectarlos construyendo un puente entre los dos ETM. Considerando el marco teórico de Duval $(1993,1995)$ relativo a la existencia de diferentes registros de representación en un solo dominio (p.e. Duval menciona el registro de números decimales de dos cifras, el de notación científica, el de números fraccionarios, todos ellos dentro del sistema de signos aritmético), y a la existencia de representaciones transitorias (DUVAL, 2006), nuestra hipótesis consistiría en la construcción de un ETM Aritmético-Algebraico $_{\text {A }}$ basado sobre contenidos matemáticos como los de incógnita, cuasi-variable, variable y covariación entre variables en un contexto aritmético-algebraico.

Bajo este punto de vista, la estructura cognitiva de los alumnos a considerar, está ligada principalmente a procesos de generalización, en donde la visualización, anticipación, sensibilidad a la contradicción, validación y prueba están íntimamente relacionados. Estos elementos nos darían a priori los primeros elementos a considerar en un pensamiento aritmético-algebraico. De acuerdo al trabajo de Saboya (2010), los elementos antes mencionados forman parte de una estructura de control sobre la actividad matemática que desarrolla el individuo.

Como lo hemos expresado antes, nosotros estamos interesados en la construcción de un pensamiento aritmético-algebraico (PA-A), ligado a un ETM $_{\mathrm{A}-\mathrm{A}}$. En la Figura 2 mostramos una adaptación del modelo de Kuzniak a nuestros propósitos. 


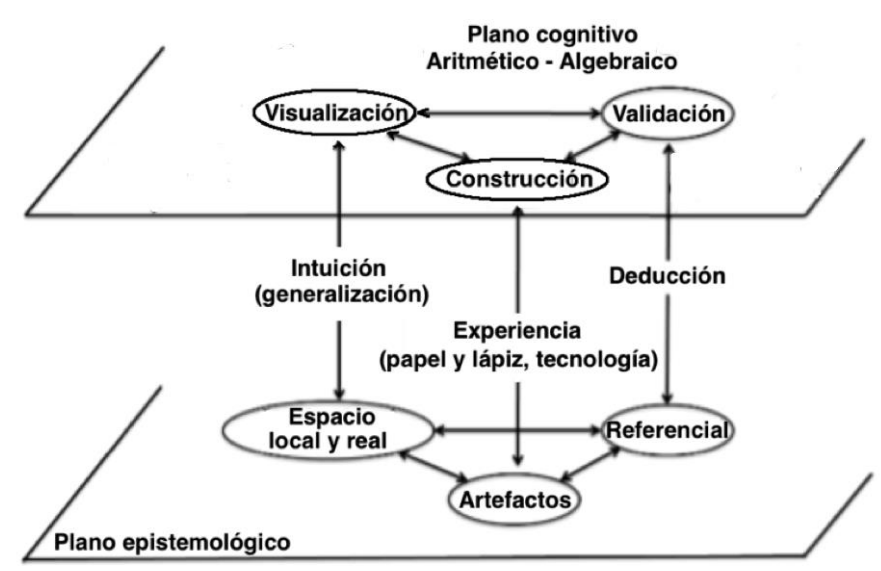

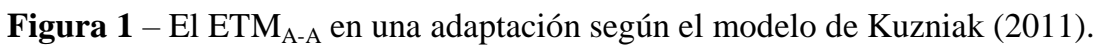

Este proceso de integración de aspectos teóricos de Duval y Kuzniak, nos servirán de base en nuestra propuesta para la creación de un pensamiento aritmético - algebraico (PA-A).

\section{Preguntas de investigación y metodología}

Una pregunta general que nos hemos planteado es: ¿El pensamiento aritméticoalgebraico se diferencia del pensamiento aritmético y del pensamiento algebraico?

A partir de esta pregunta general, hemos diseñado preguntas específicas de investigación. En un proceso de construcción sociocultural del aprendizaje ligado a un pensamiento aritmético-algebraico,

1. ¿promueve la resolución de tareas sobre generalización de patrones la construcción de un pensamiento aritmético-algebraico?

2. en los procesos de generalización de patrones, ¿qué papel juega la visualización, la anticipación, la sensibilidad a la contradicción, la validación?

3. en los procesos de generalización de patrones, ¿cómo los alumnos modelan la situación a través de expresiones algebraicas?

El método de enseñanza que hemos elegido tiene que ver con ACODESA, el cual toma en cuenta el acercamiento de construcción sociocultural e individual-social del aprendizaje, el método de enseñanza que se puede realizar con ACODESA (ver PAEZ, 2004; HITT, 2007; HITT; CORTÉS, 2009; HITT; GONZÁLEZ-MARTIN, 2015). Este método de enseñanza considera 5 etapas: el trabajo individual (en un contexto social del aprendizaje), trabajo en equipo, debate en el aula, auto-reflexión y proceso de institucionalización. Es una integración de un aprendizaje en colaboración (PAINTZ, 2001), un debate científico a la 
manera de Legrand (2001) y de auto-reflexión de acuerdo a las ideas de Hadamard (1945) y de Sfard (2008). Un resumen del método de enseñanza ACODESA se presenta en la Figura 2. Como lo muestra esa figura, en las primeras etapas, el profesor es un guía y solo es en la etapa de institucionalización que el profesor presenta los resultados utilizando representaciones oficiales ligadas a los registros de representación, haciendo énfasis en las producciones de los estudiantes. A continuación se presentan las etapas y su relación con el tipo de producción esperada:

1. Trabajo individual (producción de representaciones funcionales y producciones semióticas asociadas para comprender la situación problema),

2. Trabajo en equipo sobre una misma situación. Proceso de discusión y validación (refinamiento de las representaciones funcionales y producciones asociadas),

3. Debate (que puede convertirse en un debate científico). Proceso de discusión y validación (refinamiento de representaciones funcionales y producciones asociadas),

4. Auto-reflexión. Regreso sobre la situación (trabajo individual de reconstrucción y auto-reflexión de lo realizado en clase).

5. Institucionalización. Utilización de representaciones institucionales por parte del profesor dentro del proceso de institucionalización de saberes.

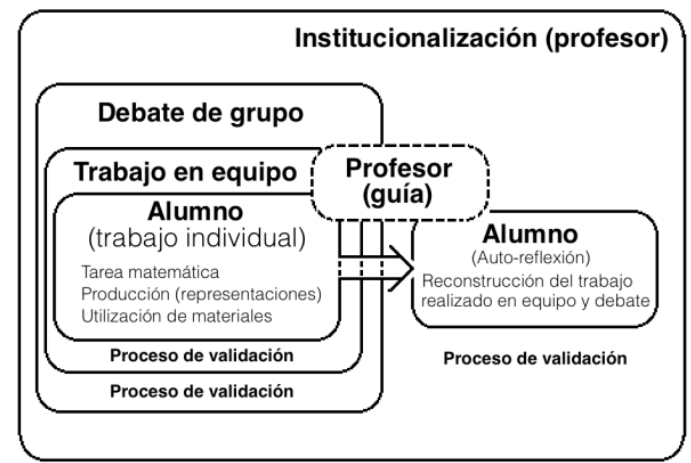

Figura 2 - ACODESA en el aula de matemáticas

\section{Diseño de actividades}

La elaboración de actividades, dentro de la metodología ACODESA, implica una estructuración de manera que favorezca la producción de representaciones funcionales, de sus correspondientes representaciones externas (representaciones espontáneas) y procesos de conversión entre representaciones. Nuestro interés en la promoción de un PA-A, nos lleva a 
considerar los patterns como el elemento clave tomando en cuenta los resultados de Kaput (2000), Kieran (2007) y reflexiones prácticas de Artigue (2012) y sobre el diseño de actividades, Prusak, Hershkowits y Schwarz (2013).

Específicamente, con el uso de patrones (patterns), nos interesa el entender cómo los alumnos construyen el siguiente término de una serie con respecto a una configuración puntual, cómo pueden generalizar a un término ligado a un número muy grande (dados los primeros elementos de una serie), cómo construir una figura dado un término ligado a un número muy grande y cómo calcular un valor determinado por la serie. Y finalmente, cómo generalizar ese cálculo a cualquier término de la serie.

Conociendo los resultados de la investigación sobre procesos de generalización con patrones en la escuela primaria e inicio de la secundaria, debemos hacer un énfasis especial en la construcción de tareas para promover el PA-A, que de acuerdo a nuestro modelo (Figura 1), consiste en promover la visualización, inferencia y uso práctico de artefactos (en nuestro caso la tecnología). Desde nuestro punto de vista, en la construcción de conceptos, todas las representaciones de los objetos matemáticos son importantes, incluyendo representaciones espontáneas no institucionales. Es decir, cada representación del objeto matemático en cuestión representa parcialmente el objeto, es por ello que la articulación entre representaciones es primordial.

Una vez que decidimos que el uso de patrones era lo más adecuado para nuestros propósitos de investigación, nos dimos a la tarea de seleccionar una actividad que pudiera promover procesos de visualización y necesariamente articulación entre representaciones. Es así que elegimos los números poligonales (desde el punto de vista numérico y figural) y relacionar esta actividad con la historia de las matemáticas relativa a esos números que se conocían desde la época de oro de los griegos.

Desde el punto de vista tecnológico y uso de números poligonales, analizamos el trabajo de Healy y Sutherland (1990) en donde se muestra que alumnos de $2^{\circ}$ de secundaria en Inglaterra llegan a producir representaciones espontáneas ligadas a procesos de iteración en el contexto de Excel: trig. $\Delta \boldsymbol{n}=\boldsymbol{n a}$ before + position . En el trabajo de Hitt (1994), él señala la importancia de proporcionar a los estudiantes otra actividad que promueva que los alumnos no queden anclados a un proceso de iteración en el contexto de Excel y puedan avanzar en procesos algebraicos que permitan calcular cualquier número triangular sin necesidad de conocer todos los anteriores. 
Todas estas consideraciones nos llevaron a concebir y diseñar actividades en papel y lápiz y uso de tecnología (Excel o calculadora y el applet POLY) en un medio de aprendizaje sociocultural en el aula de matemáticas. Este applet (CORTÉS; HITT, 2012) permite calcular el número poligonal deseado y propone una representación visual (ver ejemplo en Figura 3).

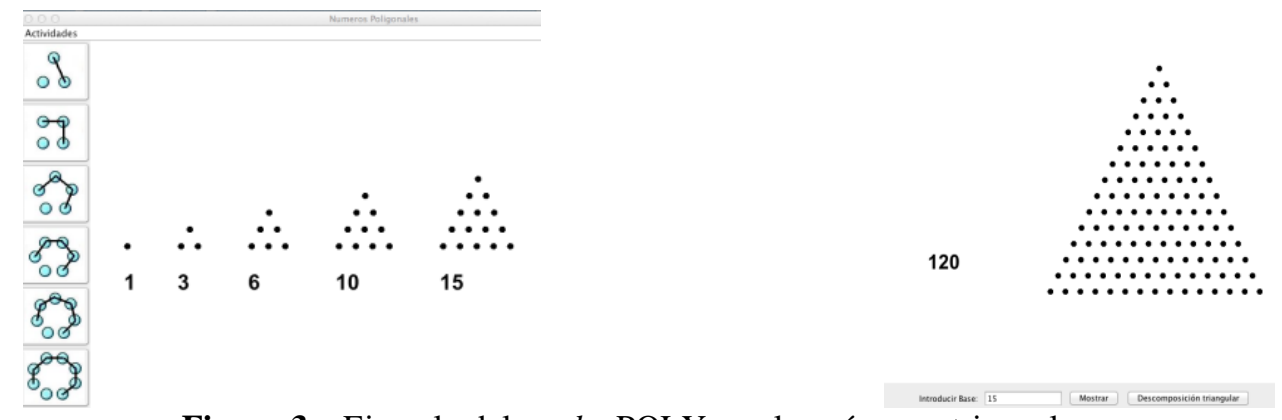

Figura 3 - Ejemplo del applet POLY con los números triangulares

En resumen, la actividad es la siguiente:

1. Se introduce la resolución de problemas en un ambiente de papel y lápiz y posteriormente tecnológico. Se explica qué son los números triangulares (desde un punto de vista histórico).

2. Se solicita calcular algunos números triangulares usando lápiz y papel. Se hace promoción de representaciones funcionales y de representaciones espontáneas asociadas.

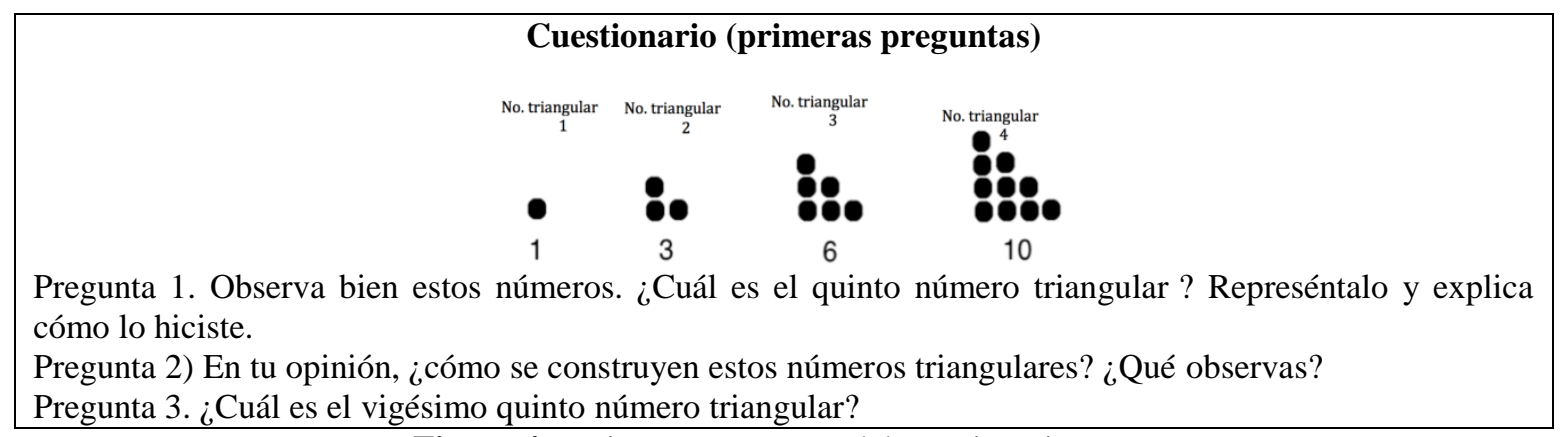

Figura 4 - Primeras preguntas del cuestionario.

Como se puede observar, las primeras dos preguntas tenían la intención de promover una estrategia ligada a aumentar un elemento en la base, en un lado, o sobre la diagonal y llenar con los puntos necesarios. Se pensaba que un cambio de estrategia se produciría al solicitar el $25^{\circ}$ número triangular, en donde seguir dibujando sería una tarea tediosa.

3. Se solicita calcular los números triangulares usando Excel o calculadora y comprobar sus conjeturas utilizando POLY. Con ello se quería promover la conjetura y prueba en el sentido de Balacheff (1997), ya que la demostración no forma parte en el nivel de estudios de estos estudiantes, 
4. Se solicitan procesos de generalización que proporcionen maneras de comunicar un resultado utilizando un número muy grande (triangular $30^{\circ}$, triangular $83^{\circ}$ y triangular $120^{\circ}$ ). Enseguida se solicita el cálculo para cualquier número. Que, de hecho, implica en cierto sentido el encontrar una expresión algebraica para cualquier número triangular. En este sentido, se promueven los procesos de generalización, predicción y conjetura. Se espera, desde un punto de vista institucional, la producción de una expresión algebraica de la forma Triangular $_{n}=n(n+1) / 2$.

\section{Resultados con la población de México}

En esta parte, presentaremos algunos resultados significativos obtenidos en México, nos interesa resaltar las estrategias y procesos de visualización que permitieron procesos para la construcción de expresiones algebraicas y del rol del trabajo en colaboración de las primeras etapas de ACODESA, así como la etapa de auto-reflexión. Nos interesa además, el papel jugado por la tecnología en el proceso de la generalización.

\subsection{Resultados obtenidos con la experimentación en México}

\subsubsection{Trabajo Individual}

En la primera fase de la metodología ACODESA el alumno de $3^{\circ}$ de secundaria trabaja de forma individual. El análisis de las producciones de los estudiantes nos permite destacar tres tipos de estrategias asociadas. En la primera, la percepción domina y las producciones están ligadas a representaciones icónicas sin contar con otro tipo de relación explícita. Como ejemplo, la Figura 5 presenta lo que hizo una alumna para calcular el número triangular $25^{\circ}$.

Figura 5 - Estrategia ligada a la percepción. 
También surgió la estrategia planificada por los investigadores (ver Figura 6) de ligar a un triángulo e ir añadiendo una bolita a la base, al lado y completar la diagonal.

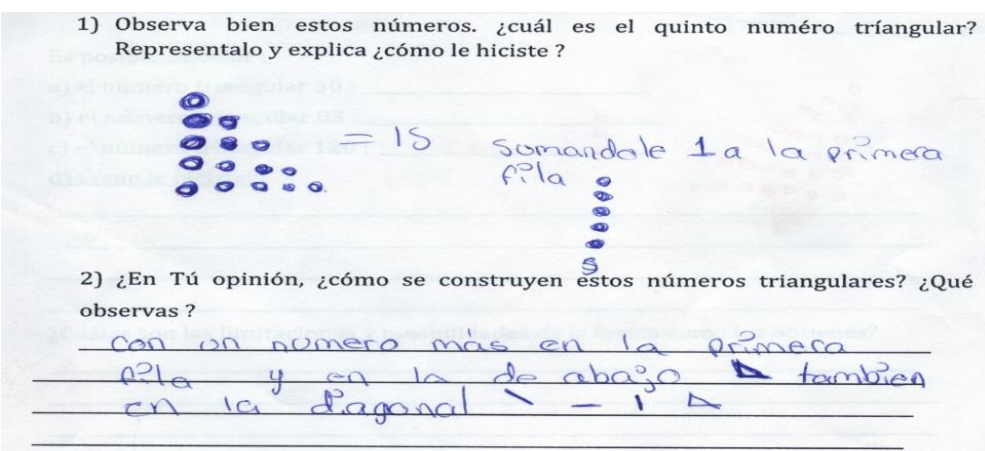

Figura 6 - Un ejemplo de estrategia en la que el proceso de visualización matemática es explícito, realizado por Karla

En la producción de la Figura 6, la respuesta del estudiante muestra que la configuración triangular juega un papel importante. Incluso, algo interesante es que en esta producción la estudiante en su mensaje utiliza representaciones no usuales para comunicar su resultado. Menciona que para construir el siguiente poligonal, hay que añadir un punto sobre la diagonal ( \) sobre la fila horizontal ( -) o sobre la fila vertical (|). Esto es a lo que nosotros llamamos representación espontánea no institucional, que está ligada a la acción de resolver una situación no conocida por el estudiante (representación funcional en la mente del estudiante ligada a la acción). Este tipo de representaciones no las podemos catalogar en algún registro de representación.

En la tercera estrategia, el alumno sigue un proceso de visualización matemática precisando que cada número triangular se puede representar como la mitad de un cuadrado (ver Figura 7). La conjetura no implica un resultado correcto, pero como el estudiante no comprobó su conjetura, no la utilizó para responder la $3^{\mathrm{a}}$ pregunta.

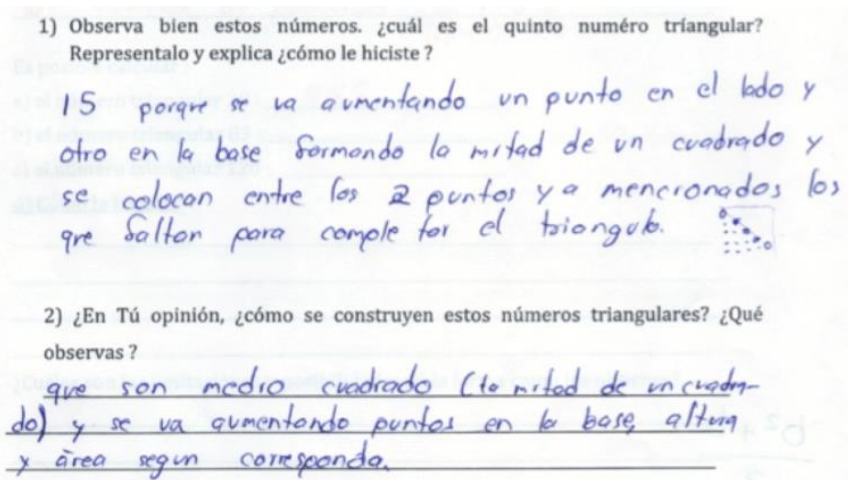

Figura 7 - Visualización, construcción de un cuadrado, realizado por Diana

En la Figura 8, se muestra otra estrategia utilizando tabla de valores. Este cambio de estrategia nos indica: 
a) Un abandono de la representación figural,

b) Un control sobre el cálculo a realizar desde un punto de vista numérico.

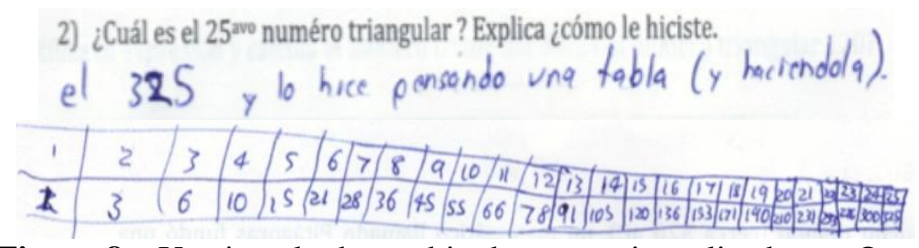

Figura 8 - Un ejemplo de cambio de estrategia realizado por Omar

En el trabajo en equipo, cada alumno proporciona su propia estrategia (correcta o no), que sufrirá modificaciones de acuerdo a la discusión entre los miembros de su equipo. En este proceso de comunicación, se espera que las representaciones funcionales evolucionen y poco a poco los estudiantes precisen sus representaciones para resolver la situación presentada.

Al contrario de lo que pasó con el grupo de Quebec con el uso de tecnología (HITT; SABOYA; CORTÉS, 2015), con el grupo mexicano, la tecnología jugó un papel menos importante. Los estudiantes se centraron en la resolución de la actividad utilizando papel y lápiz y movilizando procesos de visualización.

\subsubsection{Trabajo en Equipo (segunda etapa)}

Se formaron cuatro equipos de tres estudiantes y uno de dos. En el trabajo individual, el estudiante Omar contaba con la estrategia de utilizar una tabla de valores (ver Figura 8). Una vez que se integró el EQ4 (equipo 4 conformado por Omar, Karla y Diana), fue notoria la influencia de Omar entre sus miembros del equipo. Así, Karla y Diana, que en el trabajo individual habían seguido un proceso figural dibujando bolitas sin utilizar tablas (ver Figuras 6 y 7), con la interacción con Omar, cambian su estrategia, pasando a una tabla de valores. Se pueden seguir las huellas dejadas por ellos, ya que la consigna en el trabajo en equipo era la de utilizar tinta roja.

No se cuenta con rastros que permita explicar de donde salió el resultado que Karla sugiere como conjetura con su interacción con Omar y Diana. Solamente aparece en el cuaderno, la expresión algebraica: $\boldsymbol{y}=\mathbf{0 . 5 2} * \boldsymbol{x}^{2}$. Además, ellos no la someten a discusión cuando se pasa al debate en gran grupo. Esta producción va en el sentido del propósito de la investigación. Aunque los procesos de validación no están claros en su documento. 


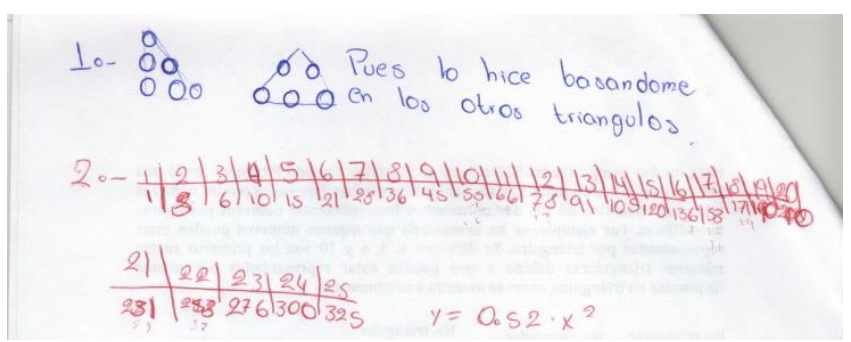

Figura 9 - Hoja de Trabajo de Karla.

Resumiendo, en esta etapa de trabajo en equipo contamos con tres aspectos:

$1^{\circ}$ La importancia del trabajo en colaboración, ya que éste promueve cambios en las ideas iniciales de los alumnos. Es notoria la influencia del estudiante Omar sobre las representaciones iniciales de Karla y Diana, promoviendo un cambio de estrategia; y a su vez, en equipo, les permitió proponer una conjetura.

$2^{\circ}$ El surgimiento de conjeturas, ya sea de tipo verbal o de tipo algebraico, como la proporcionada por Karla en su interacción con Omar y Diana: $\boldsymbol{y}=\mathbf{0 . 5 2} * \boldsymbol{x}^{\mathbf{2}}$.

$3^{\circ}$ Según lo previsto en el diseño de la actividad, proponer el uso de lápiz y papel promovería diferentes tipos de estrategias no directamente ligadas a la iteración como en el caso de Healy y Sutherland (1990).

Estos cambios de estrategia nos proporcionan elementos de reflexión sobre los procesos de visualización matemática donde la articulación entre las representaciones espontáneas, numéricas y algebraicas está en el corazón mismo de la actividad de comunicación entre los estudiantes. Específicamente, en el equipo de Karla, han pasado de la representación figural a la numérica y ello les ha permitido anticipar y, a su vez, proporcionar una conjetura de corte algebraico.

\subsubsection{Trabajo en Grupo (tercera etapa)}

En el trabajo con todo el grupo, la fórmula ligada al área de un triangulo fue la primera que se presentó por el equipo que pasó al pizarrón y la discusión se generó a partir de esta fórmula. Como lo concebimos en el diseño de la actividad, la forma de los números triangulares en forma de triángulo rectángulo hizo que los alumnos propusieran una fórmula ligada al cálculo del área presentado.

Mónica, frente al pizarrón, ejemplifica el cálculo del número triangular $8^{\circ}$. La Figura 10 presenta lo que ella realizó. Mónica menciona que para calcular el $8^{\circ}$ número triangular:

Mónica: $\quad$ Es que sacaremos lo que es el área del triángulo, que sería 8 por 8 , que son $64, \mathrm{y}$ lo dividimos entre 2 es $32 \ldots$. 
Se oye una voz muy baja de una niña que dice pero no, ¿no sería 36 ? [Dirigiéndose a sus compañeros de al lado]

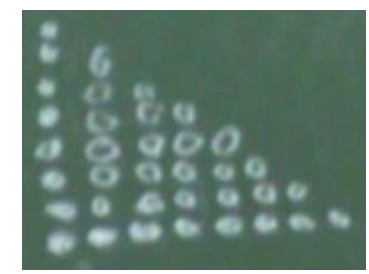

Figura 10 - Primer cálculo ligado al área del triángulo formado con 8 bolitas en la base (trabajo de Mónica)

En el análisis de la grabación, pudimos detectar que otra alumna de la clase (nos se pudo saber quien era en su intervención espontánea fuera de la cámara) había detectado una contradicción entre el cálculo numérico y lo presentado en la Figura 10 (contradicción cognitiva, ya que no es el profesor quien la ha señalado). Podemos destacar que el cálculo numérico o la representación figural de los números triangulares proporcionó una estructura de control que permitió evidenciar a la estudiante la contradicción (o ambos). Los alumnos se apoyan entonces sobre este ejemplo genérico (el octavo número triangular, intentando acomodar la fórmula para obtener los 36 puntos esperados en vez de los 32 obtenidos numéricamente.

A continuación mostramos dos episodios en los cuales el grupo de estudiantes llega a construir la fórmula final.

\subsubsection{Episodio 1 : Primera conjetura hacia la fórmula general}

\footnotetext{
Mónica: $\quad$ Es que sacaremos lo que es el área del triángulo, que sería 8 por 8, que son 64, y lo dividimos entre 2 es 32.

[El investigador escuchó a la alumna e intervino. Pero en lugar de solicitar a la alumna que explicara, mencionó lo siguiente:]

Inv: A ver, revisa esa teoría que me estás diciendo con un número triangular que tenga... [el investigador es interrumpido por Roberto]
}

Observación: felizmente, el alumno Roberto interrumpió al investigador, proporcionando argumentos propios de los alumnos. Es decir, el experto (investigador o profesor), sabe de la contradicción formal, pero es importante que sean los alumnos quienes deben detectar la contradicción (contradicción cognitiva).

Roberto: ...Pero..este, el número triangular del 8 sería 36 y no 32, entonces no puede ser.

Inv: Pasa por favor al pizarrón Roberto.

El equipo de Roberto pasa al pizarrón y va a calcular el $6^{\circ}$ número triangular. Ese número triangular había sido dibujado en el pizarrón al principio de la discusión en grupo y 
por eso lo retoman. María, del equipo de Roberto, empieza a hacer la figura escribiendo 6 bolitas en la base y 6 bolitas en la altura (Figura 11).

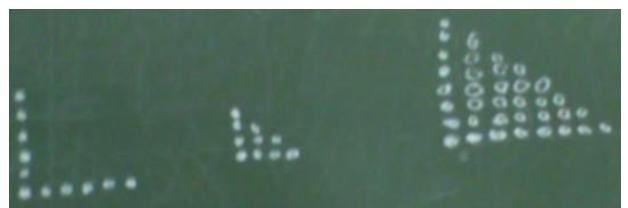

Figura 11 - Cálculo del $6^{\circ}, 4^{\circ}$ y $8^{\circ}$ número triangular realizado primeramente por María y luego por Roberto

Roberto: $\quad$ Este es el número 6 [señalando la figura que acaba de realizar María], aplicamos la fórmula que dice base por altura dividido entre; dos (ver Figura 12) sería 6 por 6, da 36, entre 2 tocaría a 18; mientras que el número triangular sería 21, entonces no daría la fórmula esta [y señala la fórmula de la Figura 12].

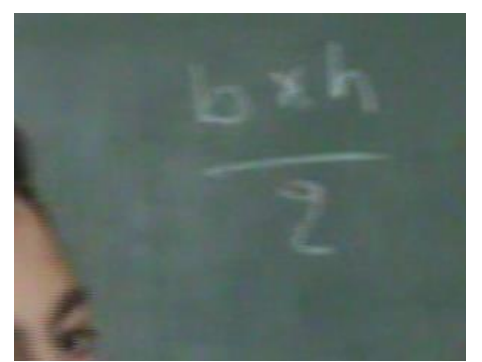

Figura 12. Formula asociada a la conjetura.

Prof: ¿Qué diferencia hay entre... ?, si aplicas la fórmula que te dice, aplica la fórmula apuntale ahí [se refiere a que escriba en el pizarrón].

María: [escribe la fórmula]

Prof. En este caso ¿cuál es la base?

Roberto: [Escribe] $6^{*} 6 / 2$ son 18 , y no es el número triangular.

Prof: ¿Cuál es el número triangular?

Los alumnos: 21 [contestan a coro]

Roberto: $\quad$ Su diferencia sería 3.

Prof: ¿Su diferencia sería...?

Roberto: 3. [Roberto está calculando el número triangular $8^{\circ}$ escribiendo la fórmula, les sale 32, ver Figura 13].

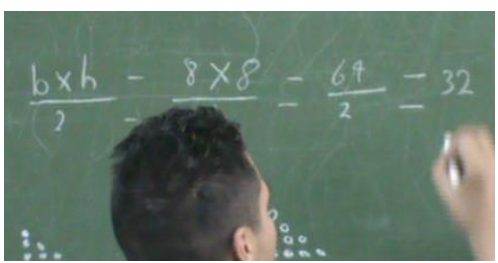

Figura 13. Contra-ejemplo, cálculo del número triangular $8^{\circ}$ para mostrar que la fórmula no funciona.

Prof: y el número triangular $8^{\circ}$, ¿cuál es?

Roberto: $\quad$ Es 36.

Prof: ¿el del $8^{\circ}$ ?

[Roberto empieza a contar las bolitas de la figura que ya tenía en el pizarrón]

Roberto: $\quad 36$ [Se oyen voces que dicen la diferencia es 4]

Observación: Cuándo el Profesor (Experto) hace la pregunta, Roberto duda y tiene que recurrir nuevamente a la estructura de control más elemental, que es contar bolitas, para 
asegurarse de que verdaderamente está construyendo un contra-ejemplo. Esto es lo que señalamos en el modelo de la Figura 1 como proceso de validación.

\subsubsection{Episodio 2: ¡EUREKA! Rectificando la fórmula en un debate científico}

El debate continúa:

Un alumno: $\quad y$ del $8^{\circ}$ es cuatro, entonces sería un medio [una voz sorprendida]

Gaby: La mitad de 8 es 4 y 4, son los números que le faltan a 32 para ser 36, entonces en la fórmula tenemos que poner base por altura entre dos, más .... [PAUSA] Más la mitad del [PAUSA] más la mitad del número triangular, la mitad [PAUSA] la mitad de la base.

Observación: Es importante señalar aquí que Gaby interviene explicitando una relación numérica y, poco a poco (en su discurso hubo varias pausas), completó su idea pasando de lo numérico a lo geométrico. El elemento de control es claro que fue proporcionado por la relación aritmética y de allí pasó a una relación geométrica (en un proceso de visualización). Pero hubo algo más, en el proceso de comunicación, al verbalizar lo que estaba pensando hubo un proceso de deducción. En estos momentos están desechando su conjetura inicial y dando pie a una nueva. Los argumentos son utilizados para refutar la conjetura.

Prof: Pasa a escribirlo..

Alumna: $\quad$ Creo que entre todos ya va saliendo algo porque de uno solo no.

Observación: La construcción sociocultural del conocimiento se ha dado en esta etapa de debate científico de acuerdo a ACODESA. La alumna expresa abiertamente la coconstrucción generada en el debate.

[Gaby pasa al pizarrón a escribir la idea que acaban de tener.]

Gaby: ¿Cómo represento la mitad de la base?

Es interesante observar que ahora Gaby tiene dificultades para transformar el argumento geométrico mitad de la base en términos algebraicos.

Inv: $\quad$... Un medio, o eso entre la mitad.

[Gaby escribe $\boldsymbol{b} \times \boldsymbol{h} / 2+1 / 2$ y entonces le dicen un medio del número triangular.]

Muchos estudiantes intervienen al unísono, proponiendo la escritura final, solicitando a Gaby de cambiar lo que había escrito. De hecho, en este proceso, Gaby se deja guiar por sus compañeros ya que ella no entiende bien el proceso de mitad de la base a la escritura $\boldsymbol{b} / \mathbf{2}$. Finalmente ella escribe: 


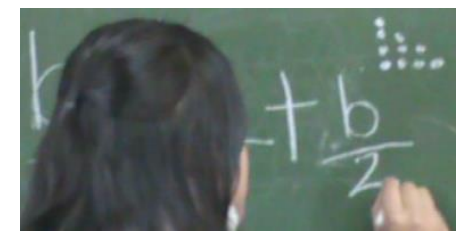

Figura 14 - Nueva conjetura, producto del debate en gran grupo (Gaby escribe)

Prof: Y ¿cómo representas el número triangular?, en esa fórmula que estas escribiendo ahí, ¿cómo representas el número triangular?

Alumnos: $\quad$.... La base o la altura...

[Gaby escribe la formula $\boldsymbol{b} \times \boldsymbol{h} / 2+\boldsymbol{b} / 2$ ]

Prof: $\quad$ Precisamente lo que él decía, la altura, a qué es igual...

Alumnos: $\quad$ A la base.

Prof: $\quad$ Entonces sustitúyela para que no metas la altura.

Alumnos: $\quad$ Sería base por base

[Gaby escribe mal la fórmula y le corrigen. y la escribe correctamente $\boldsymbol{b} \times \boldsymbol{b} / \mathbf{2}+\boldsymbol{b} / \mathbf{2}$.]

Prof: Ok, entonces, base por base ¿cuanto es?

Alumnos: Base cuadrada.

[Gaby escribe finalmente la fórmula $\boldsymbol{b}^{2} / 2+b / 2$.]

Prof: ¿Ustedes creen que esa es la fórmula ?, pruébenla con el número triangular $15^{\circ}$.

Gaby: Tengo que hacer bolitas...

Prof: $\quad$ No, no, no ya tienes la fórmula.

Observación. El profesor tiene una tendencia a desechar las representaciones figurales una vez que ya se ha obtenido la fórmula. En este caso, podemos ver una distancia entre los propósitos de los investigadores y el profesor en acción. Nuestra intención es dejar a los alumnos libremente para que sean ellos los que decidan en qué momento abandonar las representaciones figurales y utilizar las algebraicas; y lo más importante, que los alumnos realicen procesos de control transitando de lo algebraico a lo numérico o figural, como en el caso de Roberto frente a la pregunta del profesor. Esto responde a nuestras expectativas generales de nuestra pregunta de investigación y sobre la importancia de la construcción de un pensamiento aritmético-algebraico que permita al estudiante transitar de un tipo de pensamiento al otro en caso de necesidad frente a la resolución de una situación.

El debate en grupo nos permite hacer resaltar que los alumnos en conjunto rechazaron su conjetura inicial, proponiendo contraejemplos, construyendo una nueva conjetura hacia la fórmula. Es notoria la articulación que realizaron los alumnos con aspectos aritméticos, geométricos y algebraicos. Esto señala precisamente el camino de nuestras hipótesis de trabajo. La construcción de un pensamiento aritmético-algebraico es esencial para la construcción del pensamiento algebraico, en donde una estructura cognitiva ligada al control es primordial. Los procesos generados por los alumnos y el análisis de los resultados proporcionan una repuesta a nuestras preguntas de investigación. 


\subsubsection{Auto-reflexión y reconstrucción individual (cuarta etapa).}

Esta etapa, que denominamos Auto-reflexión, consistió en volver a aplicar la hoja de trabajo a cada uno de los participantes. La cuarta etapa se realizó 30 días después de las primeras tres; la asistencia fue solamente de 10 estudiantes de entre los 14, la idea principal de esta etapa dentro del proceso de enseñanza utilizando ACODESA consiste en auscultar sobre lo que el estudiante retiene después de pasar por varias etapas en donde la construcción pasó por un trabajo en colaboración.

Se les solicitó a los alumnos la realización de la siguiente tarea en forma individual:

$1^{\text {a }}$ Pregunta: Calcula el número triangular $27^{\circ}$.

$2^{\text {a }}$ Pregunta: Escribe la fórmula para calcular cualquier número triangular.

$3^{\mathrm{a}}$ Usando tu fórmula, calcula el número triangular $313^{\circ}$.

Los resultados de los 10 estudiantes que participaron en la $4^{\mathrm{a}}$ etapa, son los siguientes:

- Dos, de entre los diez, continuaron con el proceso de dibujar bolitas. Ello indica un retroceso a su posición original antes de las discusiones en equipo y en gran grupo.

- Cuatro, de entre los diez, reconstruyeron una expresión similar a la primera conjetura escribiendo: $\boldsymbol{b} \times \boldsymbol{h} / \mathbf{2}$.

- Un alumno pudo reconstruir la fórmula, pero confundió el resultado de calcular el número triangular $27^{\circ}$ con encontrar un triangular que proporcione 27 como resultado y el más próximo es el $7^{\circ}$ número triangular, que es 28 . ¡Posiblemente contó mal el número de bolitas! Su estrategia la siguió en el cálculo del número triangular $313^{\circ}$ (ver Figura 15).

- Tres reconstruyeron la fórmula exhibiendo un conocimiento estable (Figura 16). 
1. Calcula el número triangular 27 ?



2. Escribe una fórmula que te permita calcular cualquier número triangular.

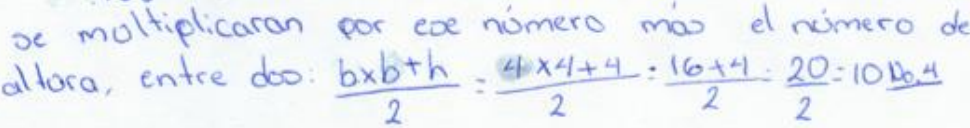

3. Usando tu fórmula, calcula el numéro triangular 313 ?

$$
\frac{24.5 \times 24.5+24.5}{2}=\frac{600.25+24.5}{2}=\frac{624.450}{2}=313
$$

Figura 15 - El alumno que reconstruye la fórmula pero confunde lo que tiene qué calcular.

2. Escribe una fórmula que te permita calcular cualquier número

$$
\begin{aligned}
& \text { triangular. } \\
& \text { Número triangular al cuadrado, entre dos } x \text { el } \\
& \text { resultado se le suma lo mitad del nomero } \\
& \text { triangular, ejemplo } \frac{(4)^{2}=\frac{16}{2}=8+2=10}{\frac{(x)^{2}}{2}+\frac{x}{2}} \\
& \text { 3. Usando tu fórmula, calcula el numéro triangular } 313 \text { ? }
\end{aligned}
$$

$$
49,141 .
$$

Figura 16-Reconstrucción correcta de la formula

Ello nos indica que, en el proceso de auto-reflexión y reconstrucción de lo sucedido en el debate, solamente tres de los alumnos realizaron una reconstrucción adecuada y uno que confundió el tipo de cálculo a realizar. El resto (seis), mostró un retroceso, regresando a su posición inicial antes del debate en gran grupo. El estudiante Roberto parece haber comprendido la idea de que su contra-ejemplo solamente lo podía validar con la representación figural. Los tres estudiantes que pudieron realizar la reconstrucción son Alejandra, Roberto y Omar. En el caso de Roberto todo parece indicar que ha construido este tipo de pensamiento PA-A. No creemos que haya sido exclusivamente con nuestra actividad, pero ella permitió corroborar la existencia y posibilidad de tal tipo de construcción.

Específicamente en las producciones que se muestran en las Figuras 15 y 16, podemos observar diferentes tipos de comportamiento:

a) Retorno a las representaciones figurales para encontrar el número triangular solicitado y errores para reconstruir o acordarse de la expresión algebraica correspondiente.

b) Alumnos que son capaces de recordar una fórmula ligada a la primera conjetura, pero que han perdido la relación con la figura. El alumno que considera el $313^{\circ}$ 
número triangular como el resultado 313 al que debe llegar y realiza un cálculo que le permite aproximarse al 313 proponiendo 24.5. Este alumno, aún cuando reconstruyó la fórmula, confundió el cálculo a realizar con los números poligonales.

c) Notación que está ligada totalmente a los procesos geométricos.

d) Notación algebraica precisa que le permite al alumno calcular el número triangular solicitado.

Estos resultados confirman la importancia de considerar una etapa de autorreflexión en el proceso de enseñanza, para poder determinar con mayor claridad los conocimientos estables en los estudiantes. Resultados similares son reportados por Thompson (2002), sobre un retroceso después de un período de consenso en la resolución de un problema. De hecho, inspirados por este tipo de resultados y de la importancia de reflexionar más tiempo sobre una actividad matemática (ver también HADAMARD, 1945) es que nosotros incorporamos la etapa de autorreflexión en el proceso de enseñanza ACODESA (HITT, 2007, 2013). Esta etapa puede proporcionarnos mayor información acerca de quiénes verdaderamente han construido un conocimiento estable.

Desde nuestra perspectiva, los resultados nos muestran que lo importante no es que resuelvan un problema, sino que se pueda construir un pensamiento aritmético-algebraico más sólido que permita desarrollar una estructura de control que sirva no solamente para aprender álgebra, sino también para aprender cualquier concepto matemático, en donde la estructura de control juegue un papel fundamental.

\section{Conclusiones}

En nuestra investigación, hemos querido presentar otra tendencia, un nuevo enfoque para estimular el debate en torno a la transición de la aritmética al álgebra basado en el fortalecimiento de la intersección existente entre la aritmética y el álgebra. Si seguimos el modelo propuesto por Kuzniak, necesitamos construir un plano cognitivamente vinculado al PA-A, que tenga un amplia intersección entre el pensamiento aritmético y el razonamiento algebraico. Por lo tanto, proponemos que en el PA-A y también en el ETM $_{\mathrm{A}-\mathrm{A}}$ tenemos que tener en cuenta:

I) La visualización matemática relacionada con la articulación entre representaciones y producción de representaciones: 
a) Reconocimiento de un patrón,

b) Visualización asociada con un algoritmo de cálculo y un proceso aritméticogeométrico,

c) Visualización de un algoritmo general aritmético-algebraico.

II) Estructura de control cognitivo:

a) La actividad debe ser presentada de manera que las conjeturas de los estudiantes puedan ser corroboradas.

b)La aritmética y representaciones figurales como una fuente de retroalimentación permanente. Por tanto, es necesario promover un proceso reversible entre el álgebra y la aritmética.

En resumen, con el enfoque presentado en este trabajo, queremos que los estudiantes se apropien del concepto de generalización en matemáticas y al mismo tiempo que desarrollen una estructura de control sobre la actividad matemática que se trate (esto podría ser importante para desarrollar una sensibilidad a la contradicción en matemáticas).

Los resultados obtenidos en la etapa de autorreflexión nos muestran la importancia de crear un conocimiento estable. Aún con todo el trabajo desarrollado por los estudiantes en cada una de las etapas, el hecho de que, un mes después de la experimentación, solo 3 alumnos de 10 hubieran podido reconstruir la fórmula exacta, muestra la fragilidad del conocimiento. Sin mencionar que algunos, en su reconstrucción, estaban en posición de contradicción y que ellos mismos no la detectaron.

La experimentación que realizamos fue corta y exclusiva de los números triangulares. De acuerdo con nuestros resultados, creemos que se debe continuar con las actividades para construir los siguientes números poligonales de forma que la actividad completa pueda proporcionar mejores resultados.

Un último señalamiento es que, de todas las representaciones que utilizaron los alumnos tanto en Québec como en México, la representación oficial $\boldsymbol{n}(\boldsymbol{n}+\mathbf{1})$ / 2 jamás la utilizaron.

\section{Referencias}

ARTIGUE, M. Enseignement et apprentissage de l'algèbre, 2012. Disponible en: < http://educmath.ens-lyon.fr/Educmath/dossier-manifestations/conferencenationale/contributions/conference-nationale-artigue-1> Consultado en: 8 Jul. 2015.

BALACHEFF, N. Processus de preuve et situations de validation. Educational Studies in Mathematics. Dordretch, v. 18, n. 2, 147-176, may. 1987. 
BROWNELL, W-A. Problem solving. In: HENRY, N. B. (Ed.), The psychology of learning (41st yearbook of the national society for the study of education. part 2). Chicago: University of Chicago press, 1942, p. 415-443.

CAI, J.; KNUTH, E. (Ed.). Early algebraization: a global dialogue from multiple perspectives. New York: Springer. 2011.

CARPENTER, T.; FRANKE, M. Developing algebraic reasoning in the elementary school. Generalization and proof. In:CHICK, H.; STACEY, K.; VINCENT, J. (Eds.). The future of the teaching and learning of algebra. Proceedings of the 12th ICMI Study Conference. Melburne: The University of Melbourne, 2001, p. 155-162.

CARPENTER, T. C.; FRANKE, M. L.; LEVI, L. Thinking mathematically: integrating arithmetic and algebra in elementary school. Portsmouth: Heinemann, 2003.

CARRAHER, D. W. et al. Arithmetic and algebra in early mathematics education. Journal for Research in Mathematics Education, Reston v. 37, n. 2, p. 87-115, march. 2006.

CHEVALLARD, Y. L'analyse des pratiques enseignantes en théorie anthropologique du didactique. Recherches en Didactique des Mathématiques, Grenoble, v. 19, n. 2, p. 221-266. 1999.

CORTES, C. ;HITT, F. POLY. Applet pour la construction des nombres polygonaux. Michoacán : UMSNH, 2012.

DUVAL, R. Sémiosis et pensée humaine: registres sémiotiques et apprentissage intellectuels. Berne: Peter Lang, 1995.

DUVAL, R. A cognitive analysis of problems of comprehension in a learning of mathematics. Educational Studies in Mathematic $s$, Dordretch, v. 61, n. 1-2, p. 103-131, feb. 2006.

DUVAL, R. Registres de représentation sémiotique et fonctionnement cognitif de la pensée. Annales de Didactique et de Sciences Cognitives. Strasbourg, v. 5, p. 37-65. 1993.

FILLOY, E ; ROJANO, T. Solving equations: The transition from arithmetic to algebra. For the Learning of Mathematics, Kingston, v. 9, n. 2, p. 19-26. 1989.

HADAMARD, J. The Psycology of invention in the mathematical field. Princeton: Princeton University Press, 1945.

HEALY, L.; SUTHERLAND, R. The use of spreadsheets within the mathematics classroom.

International Journal of Mathematics Education in Science and Technology, Philadelphia, v. 21, n. 6, p. 847-862. 1990.

HITT, F. Visualization, anchorage, availability and natural image: polygonal numbers in computer environments. International Journal of Mathematics Education in Science and Technology, Philadelphia, v. 25, n. 3, p. 447-455. 1994.

HITT F. Utilisation de calculatrices symboliques dans le cadre d'une méthode d'apprentissage collaboratif, de débat scientifique et d'auto-réflexion. In : BARON, M.; GUIN, D. ; TROUCHE, L. (Ed.). Environnements informatisés et ressources numériques pour l'apprentissage. Conception et usages, regards croisés. Paris: Éditorial Hermes, 2007, p. 65-88. 
HITT F.; CORTES C. Planificación de actividades en un curso sobre la adquisición de competencias en la modelización matemática y uso de calculadora con posibilidades gráficas. Artículo por invitación, Revista Digital Matemática, Educación et Internet, San José, v. 10, n. 1, p. 1-30. 2009.

HITT, F. Théorie de l'activité, interactionnisme et socioconstructivisme. Quel cadre théorique autour des représentations dans la construction des connaissances mathématiques ? Annales de Didactique et de Sciences Cognitives, Strasbourg, v. 18, p. 9-27. 2013.

HITT, F.; GONZALEZ-MARTIN, A. Covariation between variables in a modelling process : The ACODESA (Collaborative learning, Scientific debate and Self-reflexion) method. Educational Studies in Mathematics, Dordrecht, v. 88, n. 2, p. 201-219, feb. 2015.

HITT, F.; SABOYA M.; CORTES C. An arithmetic-algebraic work space for the promotion of arithmetic thinking: triangular numbers. ZDM Mathematics Education, Dordrecht, DOI 101007/s11858-015-0749-5, 2015.

KAPUT, J. Transforming algebra from an engine of inequity to an engine of mathematical power by algebrafying the k-12 curriculum. National Center for Improving Student Learning and Achievement in Mathematics and Science. Dartmouth, MA. (ERIC Service No. ED 441 664). 2000, p. 1-20.

KAPUT, J. J. What is algebra? What is Algebraic Reasoning? In: KAPUT, J.;CARRAHER, D., BLANTON, M. (Ed.). Algebra in the Early Grades. New York Routledge, 2008. p. 5-17.

KIERAN, C. Learning and teaching algebra at the middle school through college levels: Building meaning for symbols and their manipulation. In: LESTER JR, F. K., (Ed.). Second handbook of research on mathematics teaching and learning. Greenwich: Information Age Publishing, 2007. p. 707-762.

KUZNIAK, A. L'espace de travail mathématique et ses genèses. Annales de Didactique et de Sciences Cognitives, Strasbourg, v. 16, p. 9-24, 2011.

KUZNIAK, A. Travail mathématique et domaines mathématiques. Revista Latinoamericana de Investigación en Matemática Educativa, México, v. 14, n. 4-II, p. 385-399, 2014.

LEGRAND, M. Scientific debate in mathematics courses. In: HOLTON, D. (Ed.). Teaching and Learning of Mathematics at University Level. An ICMI Study. Utrech: Springer, 2001. p. 127-135.

MONTOYA, E. ; VIVIER, L. Les changements de domaine dans le cadre des espaces de travail mathématique. Annales en Didactique et de Sciences Cognitives, Strasbourg, v. 19, p. 73-101, 2014.

PAEZ, R. E. Procesos de construcción del concepto de límite en un ambiente de aprendizaje cooperativo, debate científico y autorreflexión. 2004. Numero de paginas/folhas. Tesis (Doctorado Didáctica de las Matemáticas). Departamento de Matemática Educativa, Cinvestav-IPN, México, 2004.

PANITZ, T. Collaborative versus cooperative learning- a comparison of the two concepts which will help us understand the underlying nature of interactive learning. 2001. Disponible en:

<http://home.capecod.net/ tpanitz/tedsarticles/coopdefinition.htm> Consultado en: 15 mar. 2016.

RADFORD, L. Gestures, speech, and the sprouting of signs: A semiotic-cultural approach to students' types of generalization. Mathematical Thinking and Learning, Philadelphia, v. 5, n. 1, p. 37-70. 2003.

RADFORD, L. Grade 2 students' non - symbolic algebraic thinking. In: CAI, J.; KNUTH, E. (Ed.).

Early Algebrization, Advances in Mathematics Education. Dordrecht: Kluwer, 2011. p. 303-322. 
PRUSAK, N.; HERSHKOWITS R.; SCHWARZ B. Conceptual learning in a principled design problem solving environment. Research in Mathematics Education, Philadelphia, v. 15, n. 3, p. 266285. 2013.

SABOYA, M. Élaboration et analyse d'une intervention didactique co-construite entre chercheur et enseignant, visant le développement d'un contrôle sur l'activité mathématique chez les élèves du secondaire. 2010. No paginas 531. Thèse (Doctorat en Education), Département de Mathématiques, Université du Québec à Montréal, Montréal, 2010.

SCHLIEMANN, A.; CARRAHER, D.; BRIZUELA, B. Algebra in elementary school. In: COULANGE, L.; DROUCHARD, J-P. (Éd.). Enseignement de l'algèbre élémentaire. Grenoble: La Sauvage, 2012. p. 107-122.

SFARD, A. Thinking as communicating: human development, the growth of discours, and mathematizing. New York: Cambridge University Press, 2008.

THOMPSON, P. Some remarks on conventions and representations. In: HITT, F. (Ed.). Mathematics Visualisation and Representations. México: Psychology of Mathematics Education North American Chapter and Cinvestav-IPN. 2002. p. 199-206.

VERGNAUD, G. La théorie des champs conceptuels. Recherches en Didactique des Mathématiques, Grenoble, v. 10, n. 23, p. 133-170. 1990.

VERSCHAFFEL, L.; DE CORTE, E. Number and arithmetic. In: BISHOP, A. J. et al. (Ed.). International handbook of mathematical education. Dordrecht: Kluwer Academic Publishers, 1996. p. 99-137.

WARREN, E. Teachers actions that assist young students write generalizations in words and in symbols. Proceedings of PME 30, Prague v. 5, p. 377-384, jul. 2006.

Submetido em Julho de 2015. Aprovado em Setembro de 2015. 